\title{
Editorial
}

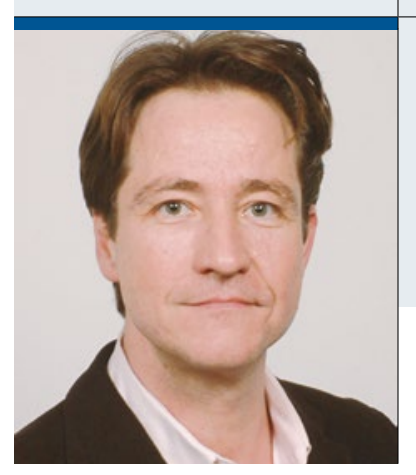

"Nach einem Schlaganfall entwickeln $40 \%$ der Patienten ein

spastisches Syndrom und 10\% chronische Schmerzen."

\section{Schlaganfallfolgen und Lebensqualität}

D er diesjährige neurologische Schwerpunkt in CardioVasc fokussiert auf wichtige und häufige Folgeerkrankungen nach einem Schlaganfall und deren Einfluss auf die Alltagskompetenz und Lebensqualität der betroffenen Patienten.

Der Beitrag von Dr. Alexander Correll widmet sich dem spastischen Syndrom nach Schlaganfall, das sich bei bis zu $40 \%$ aller betroffenen Patienten im Verlauf entwickelt. Die damit einhergehende motorische Funktionsbeeinträchtigung führt zu einer Reduktion der Alltagskompetenz und messbaren Verschlechterung der Lebensqualität der Patienten. Trotz dieser erheblichen Konsequenzen werden die vorhandenen medikamentösen und nicht medikamentösen Behandlungsmöglichkeiten nur bei etwa einem Viertel aller betroffenen Patienten konsequent angewendet. Hier besteht großer Nachholbedarf, betroffene Patienten und behandelnde Ärzte über die Notwendigkeit und die Möglichkeiten einer Behandlung des spastischen Syndroms umfassend zu informieren.

Im Artikel von Dr. Steven Drahn geht es um chronische Schmerzen, die bei etwa jedem zehnten Patienten nach einem Schlaganfall auftreten und ebenfalls von erheblicher Bedeutung für die Lebensqualität und die Alltagsfunktionalität sind. Neben der Präsentation aktueller medikamentöser und interventioneller Therapiemöglichkeiten wird deutlich gemacht, dass zunächst die Abgrenzung eines zentralen Schmerzsyndroms von anderen Ursachen notwendig ist. Die anschließende Behandlung ist dann häufig eine interdisziplinäre Aufgabe unter Beteiligung von Neurologen, Schmerztherapeuten und gegebenenfalls auch Psychiatern.
Im Beitrag von Dr. Sven Lerch geht es um epileptische Anfälle als eine häufige Komplikation nach Schlaganfall. Initial erfolgt die Diagnosestellung nicht selten verzögert, da die akute Zustandsänderung eines Schlaganfallpatienten eine Reihe von Differenzialdiagnosen aufwirft und selbst für medizinisches Fachpersonal die Einordung als epileptischer Anfall schwierig sein kann. Eine rasche Diagnosestellung ist jedoch wichtig, da häufig eine langfristige antiepileptische Therapie notwendig ist, um den Patienten vor weiteren Anfällen und den sich daraus ergebenen Komplikationen zu schützen. Es wird auch deutlich, dass bei der Auswahl eines geeigneten Antiepileptikums die häufigen vaskulären Komorbiditäten (Niereninsuffizienz!) und das Interaktionspotenzial mit anderen nach einem Schlaganfall eingesetzten Medikamenten beachtet werden müssen.

Ihr

Florian Masuhr 